\title{
1. Educación Secundaria para personas ADULTAS. UN RETO PARA EL SIGLO XXI
}

\author{
M. ${ }^{\text {a }}$ Carmen Ricoy Lorenzo
}

Facultad de Educación

UNED. Madrid.

Tiberio Feliz Murias

Centro Público de Educación Permanente de Adultos Radio ECCA. Ourense

\author{
"Toutes choses sont dites déjà; \\ mais comme personne n'écoute, \\ il faut toujours recommencer."
}

André GIDE

\section{INTRODUCCIÓN}

Distintos autores han considerado que las ideas de los llustrados del siglo XVIII sobre la educación como el instrumento esencial del progreso fueron el embrión de la Formación de Personas Adultas. De lo que no hay duda es de la fuerza y credibilidad que se tiene de la misma en la actualidad. Todo ello, su pasado y presente, estaba y está rodeado de un ideal del ser humano que pretende mejorar la situación existencial.

En la sociedad actual, a diario nos encontramos envueltos/as en constantes transformaciones, casi sin quererlo; no obstante, como seres humanos en continua evolución y cambio precisamos progresar en nuestra situación personal, socio-laboral, cultural, económica, etc. Hechos que nos llevan a hacer explícitas una serie de necesidades y a mostrar pequeñas o grandes expectativas en el día a día.

El milenio que pronto termina ha sido el milenio de la enseñanza institucionalizada generalizada desde la infancia a la adultez y para ello fueron importantes, aparte de otros muchos, los esfuerzos de los Ilustrados con relación a la Instrucción Pública que habían pensado, en su momento, en garantizar el derecho a una educación elemental común a todos/as los/as ciudadanos/as. Con sentido práctico, ésta hacía hincapié en los saberes técnicos como vía para superar la desastrosa situación en la que se encontraba el 
Estado Español en aquel momento. Se veía la enseñanza como un instrumento que podía contribuir a las adaptaciones, rupturas y transformaciones, aspectos que también hoy podemos compartir.

Un paso significativo y desde luego determinante para la Educación de Personas Adultas (EPA) parece que lo ha sido el Rapport de Condorcet, ya que se considera a éste como el primer documento legal en el que el poder político se expresa sobre la enseñanza de adultos. El sentido proselitista del cristianismo había generado la necesidad de organizar y de transmitir el saber sagrado. Así las comunidades eclesiásticas se convirtieron en centros de formación. Ante esta tradicional vinculación de la educación a la iglesia, filósofos/as y enciclopedistas proclaman que la educación ha de preparar al ciudadano/a para una sociedad civil que exige una alfabetización universal y gratuita como derecho de todos los ciudadanos/as (F. Gómez de Castro, 1988).

No obstante, en el Estado Español habrá que esperar hasta el siglo XIX para institucionalizar la enseñanza. Especial referencia de este hecho es la Ley Moyano de 1857, primera ley general del sistema educativo español que establece la obligatoriedad de la enseñanza de los 6 a los 9 años, pero no su gratuidad. En cuanto a la educación de adultos, la Ley Moyano (art. 106) hace referencia a la educación para aquellos adultos cuya instrucción haya sido descuidada, estableciendo por parte del gobierno lecciones en pueblos de más de 10.000 habitantes (art. 107). Éstas tenían que ser nocturnas o en domingo y, desde luego, aprovechando los espacios de otros locales o las propias escuelas de niños/as y sin un profesorado especializado. Herencia que ha pesado y sigue pesando sobre los/as actuales Formadores/as de Personas Adultas y consecuentemente sobre existencia de una metodología específica en/para la FPA. Podemos decir desde la distancia en el tiempo que la medida no era suficiente en aquel entonces por la enorme tasa de analfabetismo existente entre la población, como tampoco lo son las actuales. En este momento, por la evolución y el crecimiento tan rápido del saber que requiere de transformaciones aceleradas en los distintos ámbitos socioculturales, económicos, políticos, tecnológicos, etc.

Bien es cierto, que, desde la Ley Moyano, se ha ido avanzando lentamente en relación con el desarrollo de la Formación de Personas Adultas. Las posteriores leyes generales del sistema educativo español, Ley General de Educación de 1970 y LOGSE de 1990, fueron comprometiéndose un poco más. A lo largo de estos años se han venido realizando, mejor o peor, experiencias en centros culturales, empresas, escuelas técnicas y profesionales, sindicatos, ayuntamientos, escuelas taller, centros de formación de personas adultas, etc. Todas ellas han supuesto logros importantes para la consolidación y el devenir de la FPA. ésta:

En el prólogo del Libro Blanco de la Educación de Adultos se nos dice que

"Está llamada a tener un floreciente futuro en los tiempos venideros y que puede representar una contribución importante al desarrollo 
cualitativo del conjunto de la sociedad". (Libro Blanco de la Educación de Adultos, 1986:5).

En nuestra opinión, la idea gozaba de buena intención; no obstante, la aportación se hace cada vez más imprescindible para el desarrollo del propio proceso de transformación de la sociedad mundial. En el mismo Libro se recoge la necesidad de superar la concepción de Educación de Adultos como conjunto de programas escolares de segunda oportunidad, tan criticado, buscando un marco más amplio e integrador de la misma recomendado insistentemente por las Organizaciones Internacionales de la UNESCO, el Consejo de Europa y la OCDE. Posteriormente será recogida en los objetivos de la LOGSE, Título III, sobre la Educación de Personas Adultas:

“a) Adquirir y actualizar su formación básica y facilitar el acceso a los distintos niveles del sistema educativo.

b) Mejorar su cualificación profesional o adquirir una preparación para el ejercicio de otras profesiones.

c) Desarrollar su capacidad de participación en la vida social, cultural, politica y económica". (Ley Orgánica 1/1990, artículo 51.2)

Este reconocimiento supone un enorme paso hacia adelante en la FPA por los constantes desafíos que debe de ir asumiendo la misma.

En consecuencia, la FPA no puede ser de ningún modo una adaptación de los programas de la educación básica obligatoria. Aspecto del que sigue cojeando la actual LOGSE, concretamente con relación a la Educación Secundaria para Personas Adultas (ESPA), descuidando, en cierta medida, las bases psicofisiológicas, socioculturales, antropológicas y biológicas del aprendizaje de las Personas Adultas, el amplio y rico bagaje de experiencias adquiridas por las mismas, así como las propias necesidades y expectativas imprescindibles para afrontar los retos del siglo XXI. Situación que, por el momento, no se ha resuelto adecuadamente.

En la actualidad, las altas tasas de analfabetismo funcional entre la población adulta registradas en décadas anteriores han disminuido en gran medida, aunque este logro no es suficiente como tal, ya que la respuesta formativa en una sociedad tan compleja tiene que ir más allá del éxito anterior, buscando una FPA que les capacite permanente para asumir los cambios acelerados que se producen en el contexto mundial. La FPA vino a llenar un importante hueco en la Formación Permanente (FP) con la capacitación y puesta al día desde los campos de la Educación Formal, No Formal e Informal; en esta última, representan un papel importantísimo los Medios de Comunicación de Masas (MCM) y la convivencia cotidiana.

La FPA jamás ha sido conceptualizada de tan amplia y variada forma ni hemos sentido tan claramente el vértigo de una acelerada realidad que nos 
contagia y nos invade de una creciente necesidad de actualización y puesta al día. De hecho, siempre ha habido procesos de aprendizaje a lo largo de toda la vida, pero no tenían el rango de formación: los gremios eran un ejemplo. Hoy, y mañana aún más, la FPA ha perdido sus límites, en el tiempo que le damos actualmente y en los ámbitos, en los espacios y en las modalidades. Nuevos tiempos en la duración y en el momento; nuevos ámbitos sin fronteras ni barreras; nuevos espacios versátiles y flexibles; nuevas modalidades tecnológicas y organizativas. La FPA está cada día mejor considerada, siendo ésta imprescindible para afrontar las pertinentes transformaciones personales y socio-laborales del próximo milenio. En consecuencia, es difícil establecer límites en la Formación de personas Adultas. En este sentido recogemos una cita de $\mathrm{R}$. Alonso en la que, al hilo de lo que terminamos de expresar, él también manifiesta:

"La idea de la educación circunscrita al primer cuarto de la vida ha quedado desfasada. No sólo se puede aprender siempre, sino que las dinámicas sociales obligan a una formación continua, so pena de marginación cultural. Las Sociedades de la Información exigen una concepción de la formación como proceso optimizante y perfectivo que comienza y termina con la vida." (Alonso, P. 1995: 404).

Si la aparición misma de la educación institucional generalizada fue un gran paso para la humanidad del presente milenio, la eclosión de su concepción permanente configurará sin duda la sociedad del milenio que estamos a punto de comenzar.

\section{LAS PERSONAS ADULTAS COMO SUJETOS DE APRENDIZAJE}

Remontándonos en la historia podemos observar que las Personas Adultas (PA) no siempre han sido consideradas sujetos de formación, ya que las etapas de aprendizaje organizado estaban claramente delimitadas y con carácter finito. Las etapas de vida de las personas eran más cortas, en este sentido ni la niñez ni la adolescencia tenían la amplitud e importancia que se le da en la actualidad y los seres humanos vivían con premura la entrada en la adultez. Esta urgencia de "hacerse adulto" venía dada por la precariedad de la situación en la que se encontraban y la obligación de "buscarse la vida".

No siempre ha existido la educación institucional. El trabajo ha sido la preocupación primera y fundamental de las personas. El campesinado de todas las épocas ha tenido que subsistir antes que nada. La misma nobleza ha fomentado desde muy antiguo los valores de la guerra o de la caza por encima del saber o de la cultura. 
Expresiones como "hacerse persona" o "estar hecho y derecho" evidencian las creencias populares sobre la temporalidad de la formación, asociada al crecimiento biológico. Si aprender fuese crecer, terminar de crecer sería dejar de aprender; de hecho, dejar de aprender suponía empezar a trabajar. ¿Acaso debemos ir más allá de nuestra propia experiencia como personas para definir la naturaleza de nuestra educabilidad adulta? La educación se hace permanente en la medida en que se admite la educabilidad fuera de los espacios institucionales, la desligamos de la educación formal, del tiempo de crecimiento.

Lo más evidente suele ser lo primero. En formación siempre ha llamado nuestra atención la falta de lo más básico. Sin duda en nuestra cultura -y pensamos que lo básico en formación está en función de los parámetros culturales e ideológicos del contexto- es preponderante la importancia de la escritura, aunque no tiene por qué serlo en todo lugar y siempre.

La propia terminología "Educación de Personas Adultas" está recogida por la UNESCO (XIX Conferencia General. Nairobi, 1976) designando a la totalidad de los procesos formativos organizados, al margen de los contenidos desde los distintos campos de la Educación Formal y No Formal, desarrollada por diferentes instituciones y en distintos lugares, afectando a los campos de la educación de base, la ocupacional, socioparticipativa y la educación para el ocio debiendo enlazarla con la educación adquirida anteriormente y las distintas experiencias, necesidades y expectativas de las PA, capacitándolas y potenciando su formación integral a lo largo de la vida. Como tal Formación Permanente que es, supondrá importantes repercusiones sobre los diferentes campos mencionados y desde los distintos niveles de la misma.

La enseñanza con PA debe responder a un proceso de comunicación bidireccional que contemple la coparticipación del alumnado y profesorado en torno a las propuestas y toma de decisiones en la adquisición de los conceptos, procedimientos, valores, normas y actitudes para la búsqueda de un progresivo aprendizaje autónomo. Pensamos que el punto fuerte del aprendizaje adulto es su propia experiencia, y en este sentido coincidimos con Jarvis en que:

"Esto llevará a una exposición del ciclo de aprendizaje en el que éste se ve como un proceso de reflexión sobre la experiencia en lugar de la memorización de datos". (Jarvis, P., 1989: 120).

El alumno adulto, en absoluto, es, como muy bien ha descrito Paulo Freire, un receptáculo vacío que necesita ser llenado con los conocimientos del profesor, sino todo lo contrario, es una persona con buenas y malas, grandes y pequeñas experiencias que, al menos éstas, suponen un bagaje real concreto y determinante en la adquisición de nuevos conocimientos de los que se ha de partir para continuar su proceso madurativo integral. El alumnado adulto debe de ser y sentirse coprotagonista de su propio aprendizaje y consecuentemente de su progresivo desarrollo psicológico e intelectual. El aprendizaje con las PA ha de tener sentido dinámico y dinamizador de crecimiento 
permanente, aunque obviamente desde la realidad misma de sus limitaciones y posibilidades.

Aparte de considerar las características psicofisiológicas, socioculturales, antropológicas y biológicas en el aprendizaje de las PA, como referentes para contribuir al desarrollo de los procesos de maduración y aprendizaje hemos de tener en cuenta las necesidades y condicionantes particulares de cada Personas Adulta en particular y del sociogrupo-clase/comunidad-, así como su potencial de aprendizaje y nivel de expectativas. El formador de Personas Adultas juega un rol importante en el conocimiento de las propias PA y en el desarrollo de sus expectativas vitales. Aspectos determinantes con relación a emprender metas y afrontar retos permanentemente.

Recogemos, para terminar este epígrafe, las características del aprendizaje adulto que J. Sarramona (1989) considera que hemos de tener en cuenta:

- Motivación intrínseca sobre la promoción/adaptación/cambios sociolaborales, y pensamos que hemos de buscar también que la extrínseca tenga un papel importante en el proceso la maduración de las PA.

- Dimensión individualizadora derivada de la propia persona que consideramos será complementada con la sociogrupal derivada del grupoaula (alumnado, profesorado) y comunidad/barrio/pueblo.

- Madurez y responsabilidad específica del "ser adulto".

- Funcionalidad que busque la propia aplicación del desarrollo madurativo a las circunstancias y expectativas personales y sociogrupales.

- Pragmatismo en la resolución de problemas cotidianos.

- Visión holística de la realidad, porque ésta no se puede dar fragmentada.

\section{EL GRADUADO ESCOLAR: UNA EPOPEYA CONCLUYENTE}

Nada volverá a ser igual. Parece el título de un poema o de un soul pero es una intuición. En la historia de España nunca una titulación había movido a tanta gente por tan distintos motivos para acudir a un Centro de Formación de Personas Adultas (CFPA). Quizá la propia eclosión de los CFPA, con todos sus condicionamientos y particularidades, y el mismo florecimiento de este sector formativo, unido a la misma superación de ese mismo corsé, estén irremediablemente ligados al Graduado Escolar. Esta titulación ha sido la semilla para la creación de la mayor parte, si no de todos los Centros de Formación de Personas Adultas bajo la primitiva denominación de Centros de Educación 
Permanente de Adultos (EPA). La demanda de esta titulación ha estado ligada, primero, a la población de cierta edad sin oportunidades para su obtención, y ha derivado con el tiempo hacia la recogida de la población más joven salida sin ella del sistema obligatorio de enseñanza. La evolución del perfil del alumnado se ha podido observar de forma reiterada en cada uno de nuestros centros.

Los propios centros han visto cómo su desarrollo rompía con sus moldes. La demanda de la población, particularmente la que había ido consiguiendo esta titulación, junto al desarrollo de una cultura, identidad y capacitación profesional de los/las docentes de estos centros ha favorecido la ampliación de las ofertas formativas y ha originado una reflexión, provocando una apertura de los horizontes a la vez que una integración de las ofertas o programas que antes se habían considerado desvinculadas de estas actuaciones, bien por no entenderlas como formación o por no valorarlas en igual medida.

El esfuerzo y la reflexión del profesorado, en su mayor parte con una formación inicial de Magisterio sin más especialización que las que disponía esta titulación, han sido el motor y el agente materializador de esta eclosión.

No obstante, nada volverá a ser igual. Y ello porque nunca se volverán a dar las circunstancias que han avocado a nuestra situación actual, fruto en gran parte de las siguientes circunstancias:

a) Las condiciones sociológicas -democracia reciente, inestabilidad de las condiciones laborales, emancipación femenina- han favorecido el desarrollo de actitudes de búsqueda para la mejora personal/familiar y socio-laboral que no se volverán a dar. En todo caso, y fruto de este avance general y básico en la formación, la demanda se está especializando y focalizando hacia intereses personalizados -académicos, profesionales o personales- aprovechando la creciente y diversificada oferta formativa para Personas Adultas. La necesidad para el acceso al mercado o a la promoción laboral ha sido la motivación más frecuente para su consecución. Además, frecuentemente se necesitaba un tiempo razonable -1 curso escolar- para su obtención. El vacío formativo difícilmente se repetirá, entre otras cosas porque el Graduado Escolar seguirá posibilitando el acceso al mercado laboral como en su momento ocurrió con el Certificado de Estudios Primarios, con la diferencia de que de éste disponían muchas menos personas.

b) Un profesorado entregado, con una formación pedagógica -aunque deficiente y generalista, pero pedagógica al fin y al cabo- que ha desarrollado una vocación, sin diseños previos, vinculada a la experiencia y al propio desarrollo profesional, entre dificultades y desaciertos, pero también logros y éxitos. Ni el profesorado que se está implicando ni el propio diseño de los centros, en proporción creciente como partes 
integradas de institutos de enseñanza secundaria, nos ofrecen las condiciones para desarrollar nuevamente esta EPOPEYA, si se nos permite un nombre honorífico.

En fin, nada volverá a ser igual. El boom de la Formación para las Personas Adultas ha ido de la mano del Graduado Escolar y ha pasado. Las demandas se han diversificado y especializado; el alumnado ha cambiado; el listón se ha elevado; y los profesionales se han formalizado. No queremos decir con ello que será peor. Algunas cosas se perderán, pero en su conjunto crecerá y tendrá que asentarse de un modo definitivo como un elemento consubstancial a nuestras vidas. Mas, al igual que recordamos el cachorro con nostalgia y entregamos nuestro afecto al perro adulto, sin olvidar nuestra fuente, debemos mirar hacia adelante asumiendo que nada volverá a ser igual... aunque tendrá que llegar a ser mejor. Es una cuestión de tiempo, trabajo constante y entusiasmo.

\section{EL DESARROLLO DIVERSIFICADO DE NUESTRA FORMACIÓN SECUNDARIA PARA PA}

Uno puede tener a veces la impresión de que avanzamos, aun siguiendo en el mismo punto, como si una fuerte inercia nos mantuviese anclados/as al muelle de partida sin dar la salida. Quizá sucede efectivamente lo que asevera André GIDE: no se escucha. Aquí los matices son múltiples y diversos: hay quien no quiere, quien no puede o a quien no dejan. En fin, como indica el título de este trabajo, lo vamos a coger con calma y pensaremos ya en el próximo siglo. Esperando que en los próximos 100 años haya quien pueda, quien quiera y a quien dejen escuchar y observar.

Empecemos por lo más básico y necesario: la formación a la que nos referimos va encaminada a la obtención del título de Graduado en Educación Secundaria, denominación que acuña la LOGSE en su capítulo III de la sección primera (Ley Orgánica 1/1990, artículo 22.2). Esta titulación se puede obtener como consecuencia de la superación del último curso de la Educación Secundaria Obligatoria (ESO), pero también como resultado de un proceso de formación post-obligatorio, es decir, no obligatorio, o de pruebas (Capítulo IV, sección primera, artículo 52). Este aspecto no es baldío. Por una parte, tiene que ser la misma titulación. El artículo 22.2 dice bien claro que "es una titulación única". No se admiten, pues, variantes ni matices en la titulación en sí misma; otro aspecto bien diferente es la forma de obtenerlo, y a ello llegaremos más adelante. Pero, además, pone de manifiesto que es una titulación que se obtiene como consecuencia de un proceso de formación. Este aspecto tan evidente y aparentemente indiscutible es uno de los lapsus que, a nuestro entender, deja la LOGSE en su definición de la estructura del sistema educativo. Se define la estructura antes expuesta y la titulación de 
GES como consecuencia de superar el $4 .^{\circ}$ curso de ESO. Los contenidos de la ESO se establecen como consecuencia de lo que se cree que el alumnado "normal" de esa etapa y en esa edad puede alcanzar con un proceso "normal" de enseñanza-aprendizaje. Ahora bien, no se define la titulación en sí, o, dicho en otras palabras, no sabríamos decir qué es un GES si no es con referencia al currículum de la ESO. No es necesario profundizar mucho para deducir que cualquier constructo curricular conducente a esa titulación dependerá por ende del currículum de la ESO, como se puede apreciar en la gráfico 1.

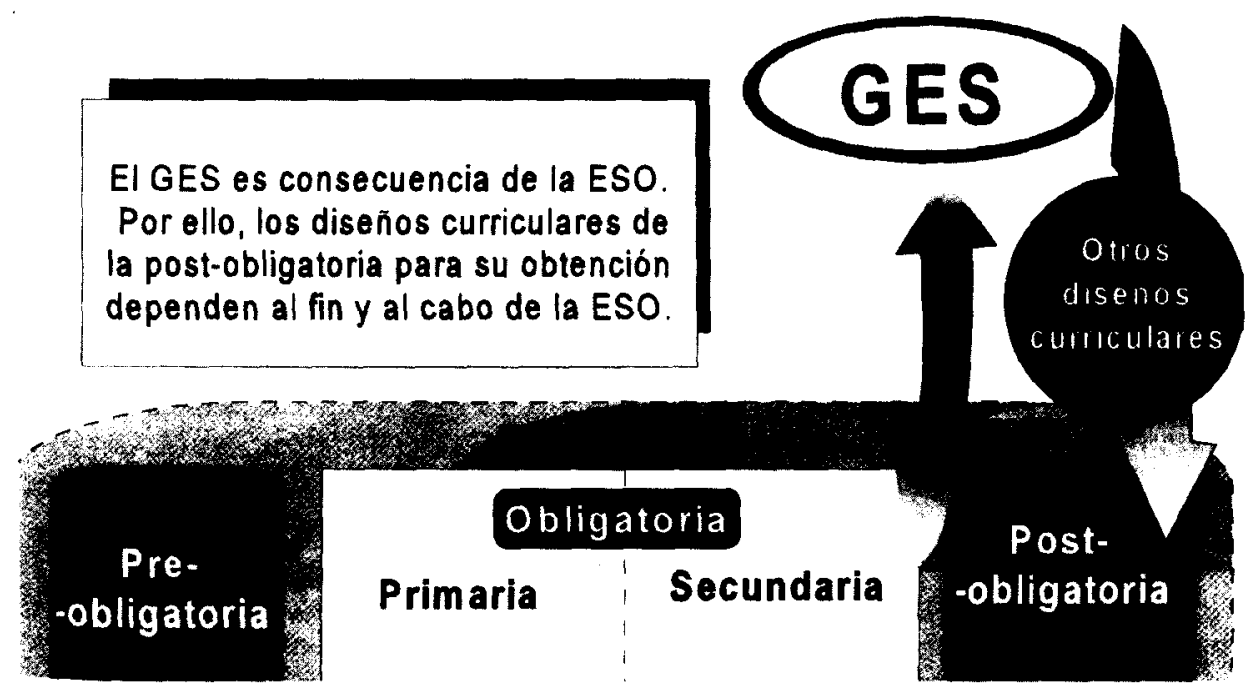

GráfICo 1. Dependencia del Graduado en Educación Secundaria de la Educación Secundaria Obligatoria.

Este tema tiene una cierta trascendencia a la hora de plantearnos diseños curriculares conducentes a esta titulación, puesto que se ha interpretado la equivalencia de currícula como una adaptación del currículum "principal", es decir, del de la ESO. Podría no haber tenido tanta si la equivalencia se hubiese entendido simplemente por el hecho de conducir a la misma titulación. Pero se ha querido entender como una adaptación del currículum de la ESO. Evidentemente, cuando hablábamos de "curriculum específico", no todos/as hablábamos de lo mismo. A todas luces, hay quien no quiere escuchar. Para la próxima reforma, cuando sea, sin prisas, podemos empezar por el principio: plantearnos una pregunta de reflexión como:

¿Qué consideramos necesario en la formación básica de un ciudadano o ciudadana en nuestra sociedad? Es decir, ¿qué necesitamos para vivir aquí y hoy? ¿Cuál es la perennidad de nuestro saber cotidiano? ¿Cómo nos actualizamos? 
Son múltiples las metodologías aplicables. Pueden resultarnos útiles la metodología Delphi (Leirman, W., 1994: 87), estudios sobre la población o el trabajo etnográfico participativo (Pérez Serrano, G., 1994). Esos "mínimos" podrían convertirse en la titulación básica del sistema educativo que se obtendría tanto al finalizar el proceso de educación básica obligatoria como a través de los mecanismos -formativos o no- previstos para Personas Adultas (véase gráfico 2). Los procedimientos de obtención del GES son equivalentes porque dan lugar a la misma titulación, no porque sean procedimientos semejantes, ni en la forma ni en el fondo.

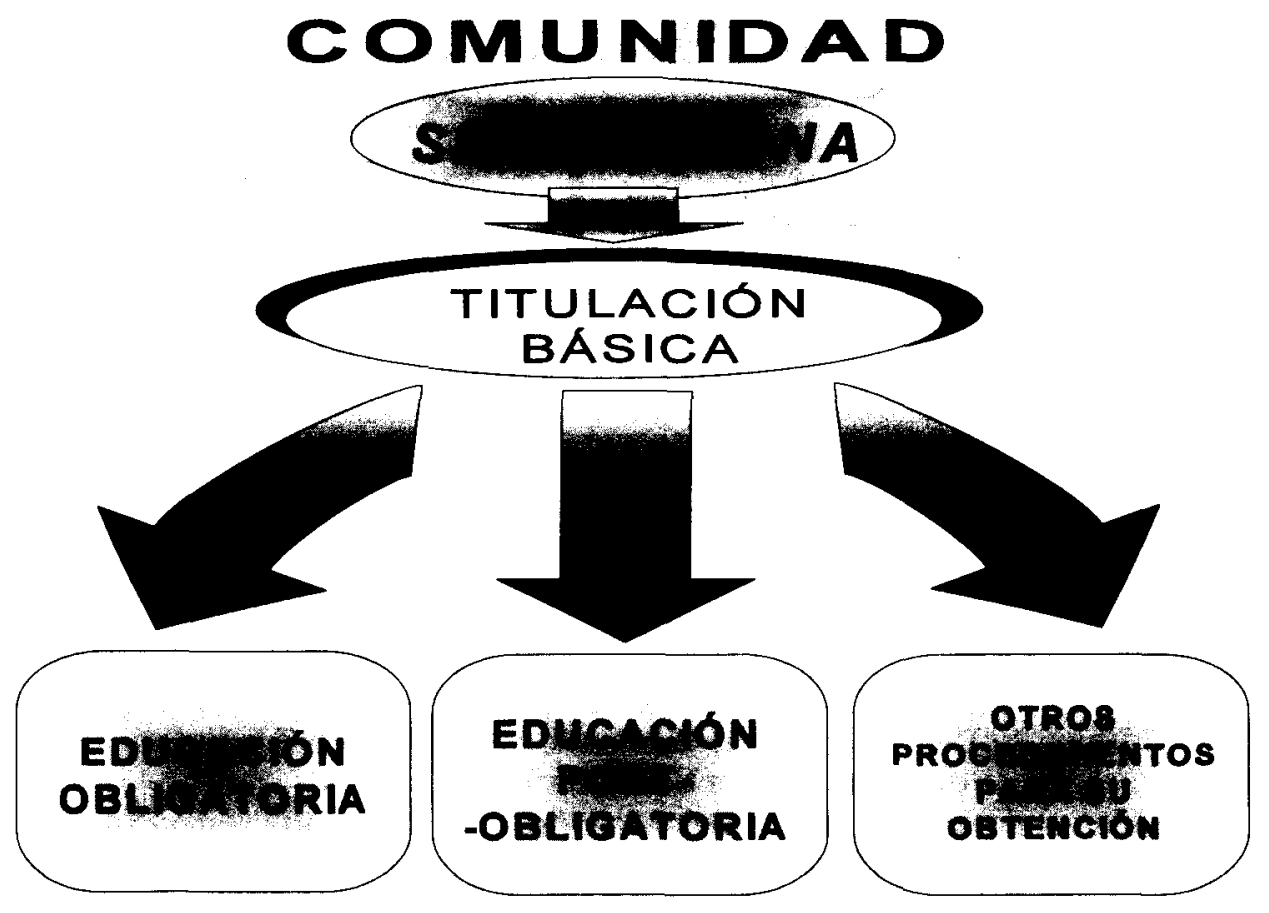

Gráfico 2. El Ser Persona en Sociedad como referencia de la Titulación Básica.

Finalmente, no debería sorprendernos la posibilidad de obtener una titulación aportando "créditos" o "valores" que incluso no tienen por qué ser formativos, sino también profesionales o simplemente vitales. La titulación básica es, desde nuestro punto de vista, una acreditación de madurez, es decir, como tanto se lleva repetido en los últimos tiempos y quizás tan poco practicado, una manifestación del "saber hacer, saber ser y saber estar" que evidentemente se pueden adquirir y demostrar por muchos medios. Desde este punto de vista, conviene restablecer el equilibrio necesario entre los subsistemas del sistema educativo de cara a centrar el reto del próximo siglo que, 
a nuestro juicio, se situará en la consolidación de la educación permanente, holísticamente entendida, en el que cada vez perderá más peso la zona obligatoria de la formación por su creciente relatividad, caducidad y obviedad. En este sentido, la dependencia del sistema obligatorio cada vez carecerá más de sentido. Estos rasgos se irán también asentando en los países menos desarrollados, aunque previsiblemente con más calma.

Una vez situada nuestra formación en el contexto educativo, tenemos que ir concretando hasta aterrizar en el aula y en cada pupitre. Los niveles de desarrollo curricular no son tan obvios. Como hemos observado en el epígrafe anterior, la movilidad ciudadana conlleva bastantes ajustes para posibilitar un ejercicio justo de los derechos personales. Analicemos al menos la casuística planteada por la laguna constitucional sobre la Formación de las Personas Adultas.

\section{MODELOS ORGANIZATIVOS EN EL ESTADO}

Como consecuencia de la Constitución de 1978, nuestro marco legislativo se estructura en un nivel Estatal y otro Autonómico antes de descender al ámbito Municipal. Ante la ausencia de una organización competencial en nuestro marco jurídico, cada Comunidad Autónoma puede establecer la organización que considere oportuna con el marco legal correspondiente. El ámbito de la Educación de Personas Adultas ha sido un ejemplo. Empezaron así a aparecer cuadros legislativos diversificados -a veces apoyados en leyesmarco, a veces no' - que auguraban realidades muy diversas. Aun admitiendo la normal transferencia de planteamientos, la diferencia se hacía notoria entre las experimentaciones en curso para el Graduado de Educación Secundaria. Por ello se iniciaron unas reuniones entre representaciones de las diversas Comunidades Autónomas con el fin de coordinar las actuaciones entre los distintos ámbitos competenciales, estableciendo así unos Acuerdos sobre los elementos comunes a todo el Estado para la elaboración de un diseño curricular específico de la Formación Básica de las Personas Adultas, en 1993. No sabemos aún en qué medida servirá este acuerdo ínter-autonómico. Una lectura de los acuerdos muestra su superficialidad e inespecificidad. Ello se explica de hecho porque se propone durante una andadura de desarrollo curricular -o al menos de proyectos en experimentación- ya avanzada que daba pie a un escaso margen de maniobra, por lo que originaba unas estrechas posibilidades de concreción. Lo más destacable de este acuerdo podría

\footnotetext{
'Mientras, por ejemplo, en Andalucía y en Galicia se aprobaban Leyes de Educación de Adultos, el propio Ministerio de Educación, responsable de las competencias en otras muchas comunidades autónomas en aquel momento, ha realizado el desarrollo normativo a través de legislación de rango menor.
} 
ser la aceptación del Currículum de la Secundaria Obligatoria como marco de referencia para el desarrollo de currícula específicos de Personas Adultas. Apréciese que esta premisa es definitoria y establece por sí sola un marco de acotación muy específico que posibilitó, en su momento, la salida a otro tipo de acuerdos mucho más complejos, aunque probablemente de mayor interés para el desarrollo de este sector formativo. Otro tipo de acuerdo hubiese pasado, por ejemplo, por establecer un mínimo común en relación con la estructura y/o el currículum -objetivos, contenidos, criterios de evaluación-, o establecer equivalencias entre los diversos modelos. No obstante, cualquiera de estas soluciones hubiese supuesto un mayor nivel de desarrollo curricular en cada una de las Autonomías participantes. Esto da pie para plantearnos los siguientes interrogantes:

- ¿Quizá debemos ver en ello un cierto temor conjunto a realizar propuestas? $¿ \mathrm{O}$, simplemente, la dejadez con que hemos vivido la reforma en la Formación Permanente de Personas Adultas en algunas Comunidades Autónomas?

Y si esto no resultase creíble, sugeriríamos una revisión de los calendarios de la experimentación de las propuestas curriculares para el GES en los centros de FPA. Por ejemplo, en Galicia se realizó la primera implantación experimental de diseño curricular en el curso 1997-1998. Por cierto, este currículum había sido elaborado durante el curso anterior y aprobado en julio de 1997. ¿Admitiríamos -profesorado, alumnado, sindicatos, partidos políticos, etc.- una improvisación tal para cualquier otro sector formativo?

Habrá que retomar este tema en alguno de los sentidos alternativos apuntados ante la necesidad de llegar a equivalencias o reconocimientos con otros países, particularmente con los de la Comunidad Europea. La movilidad ciudadana engendra estas necesidades. No es menos probable que, en un futuro inmediato, -si es que no nos es válido ya- se produzca una mayor movilidad migratoria entre los territorios fronterizos de países diferentes antes que entre territorios alejados dentro del mismo país. Así, en estos momentos, se están instalando enfermeros/as gallegos/as en el norte de Portugal ante la fuerte demanda que se produce en ese país y algunos/as estudiantes de enfermería han optado por realizar su formación en el país vecino en presencia de las dificultades que encuentran en el nuestro para ello. Y el futuro acrecentará estos fenómenos hasta su total normalización.

La ruptura de los límites también ha afectado a las modalidades de formación. En efecto, la clasificación entre educación presencial y a distancia, a veces matizada con la semi-presencial, resulta más una distinción académica -entiéndase desde el punto de vista de las Ciencias de la Educaciónque práctica. La misma diversidad de actuaciones dificulta una clasificación adecuada de las modalidades, además de evidenciar su ineficacia y su inutilidad. Resultaría tal vez de mayor interés la consideración de un gradiente de análisis que permitiese caracterizar las diversas modalidades de actuación 
entre 2 polos extremos -presencial y a distancia- en función de diversos parámetros tecnológicos -en sentido amplio- como los:

- Recursos didácticos

- Medios tecnológicos

- Formas de aprendizaje

- Contacto con el profesorado

- Medios y modos de evaluación, entre otros.

Este símil procede del campo de la física para expresar magnitudes comparables entre sí, pero no acumulables (piénsese en la temperatura, por ejemplo); nuestro afán no es medir sino caracterizar. Resulta, a nuestro entender, de mayor interés un modelo caracterizador que clasificador en un panorama tan vasto y diverso. Podemos observar que cualquier modalidad de formación tiene aspectos presenciales y a distancia, es decir, momentos de contacto con los/las formadores/as y momentos de trabajo autónomo en su hogar. Revisando las modalidades que conocemos en nuestro entorno, nos daremos cuenta de que esta graduación variable -por ejemplo en función de las edades, del estilo del profesorado o de los niveles académicos- se da incluso en el sistema formal de educación obligatoria. Esta variedad es sin duda mucho mayor en la FPA. Por otra parte, la misma división presencial/a distancia, entendida dicotómicamente, suscita barreras difíciles de conjugar:

- ¿El trabajo a distancia es siempre autónomo? ¿El trabajo presencial es siempre dirigido?

- ¿En qué medida se asemeja más el trabajo autónomo en el aula, basado en programas informáticos de autoaprendizaje, por ejemplo, con el trabajo a distancia "stricto sensu", antes que con la tradicional clase expositiva de toma de apuntes, o ésta con una clase radiofónica estilo Radio ECCA en que se emiten por radio explicaciones de formadores/as que siguen fichas escritas mutiladas -"esquemas"- que se van leyendo, completando y explicando?

No hace falta profundizar mucho para ver que las modalidades formativas tienen que tender a diversificarse y que los avances técnicos crearán su propia diversificación que, de hecho, ya ha comenzado. La modificación de cada una de las variables antes apuntadas, y que podríamos considerar sobre un continuo bipolar -o de más polos-, dará lugar con certeza a todo tipo de modalidades formativas todavía inimaginables.

También irá cambiando la concepción de las instituciones formativas. En la FPA se está ya percibiendo una gran variedad de instituciones, empresas y grupos que acceden a programas de formación. En el futuro la lectura se hará 
por defecto: no habrá instituciones, empresas o grupos que no participen de algún modo en uno o varios programas/modalidades/campos de actuación de la FPA. Esta afirmación se puede realizar sin riesgo alguno en el campo no formal; en el campo que nos ocupa, será de interés que se sigan desarrollando programas de mayor versatilidad que los centros estables para atender necesidades puntuales o prioritarias. Ello no es óbice para que las comunidades autónomas piensen en desarrollar una red territorial estable de centros/espacios de FPA, posiblemente integrados en espacios más abiertos de animación sociocultural o de desarrollo local que coordinen, promuevan y estimulen las acciones sobre/de una población territorialmente definida. En cualquier caso, es necesario y conveniente que se siga estimulando la participación de grupos, empresas o instituciones.

\section{EL FUTURO PASA POR LA FLEXIBILIZACIÓN}

En este contexto, el sistema modular viene constituyendo una propuesta de organización que, desde diversos tratamientos, da forma al currículum de GES. Pero por lo de ahora, lo que se ha visto se parece más a una compartimentación que a una modularización: se ha convertido la unidad del curso escolar con todas sus connotaciones -matrícula, organización, programación, evaluación- en 2 sub-unidades cuatrimestrales, que de modular sólo tienen el nombre. Pasa con estos sistemas "modulares" que la moda los reduce al más simple formalismo y, queriendo flexibilizar los procesos, los petrifican e impiden siquiera un elemento básico de la flexibilización didáctica, tal es el ritmo de aprendizaje, al tiempo que vulneran los principios más básicos sobre los procesos de orientación al posibilitar el reconocimiento de un nivel, independientemente del que realmente tenga en ese momento. Si de algo debemos aprender en estos años constituyentes de la FPA es precisamente de la importancia -determinante y definitoria- de la diversidad. La diversidad no tiene que servirnos para uniformizarla, sino para respetarla y asumirla como característica intrínseca de nuestra acción; tiene que llevarnos a un principio de organización consubstancial en la FPA: la flexibilización. Debemos, pues, estar abiertos a todas las posibilidades que podamos ofrecer en función de los medios de que dispongamos.

Nos quedaría por afrontar la posibilidad de la vulneración del derecho a la igualdad de oportunidades. Pero creemos que en la uniformidad se vulnera con mayor facilidad porque: ni es más justo dar a todos/as por igual, ni exigir a todos/as lo mismo, ni omitir las particularidades de cada cual. La flexibilización se asienta en diversos principios:

- Debemos aceptar nuestra actuación en y con la diversidad. Hablar de diversidad es bien diferente cuando el alumnado forma un grupo de coetáneos/as a cuando se conforma con PA. 
De hecho, esta complejidad se refleja en la escasez de autores/as que han estudiado la psicología evolutiva adulta y los/as grandes investigadores/as de este campo lo han omitido o reducido ante esta dificultad. La definición del GES tiene que admitir diferenciaciones más allá de las que podría permitir para los/las jóvenes de nuestra sociedad durante su formación obligatoria. De hecho la LOGSE, al permitir las diversificaciones del currículum en las enseñanzas mínimas para mayores de 16 años en centros ordinarios, establece que:

"En este supuesto, los objetivos de esta etapa se alcanzarán con una metodología especifica, a través de contenidos e incluso de áreas diferentes a las establecidas con carácter general." (Ley Orgánica 1/1990, artículo 23.1).

Este supuesto debería hacer reflexionar a los/las profesionales y administradores/as. Si somos capaces de asumir esta flexibilidad para los centros ordinarios, con una variación máxima en las edades de 2 años, ¿por qué empeñarnos en no aceptar tal grado de posible diversificación entre las PA?:

- Debemos ser sumamente respetuosos con las opciones personales. Las PA -aun las más jóvenes- son personas muy conformadas en experiencias -que las determinan-, compromisos -que las obligan- y expectativas -que las dirigen-. Todas ellas son respetables, dentro de nuestro marco democrático. Por ello, la titulación básica no puede configurarse independientemente de y con la PA. Ello significa que cualquier currículum debe ser personalizado y negociado con el/los sujeto/os que lo va/n a realizar. La negociación es un aspecto crucial en la FPA (Sepúlveda, F., 1997). También lo deben ser la opcionalidad, la optatividad, la propuesta personal y la autodiversificación curricular. Nuestras aulas se tendrán que parecer más a salas de estudio o de investigación con espacios para el trabajo autónomo y el trabajo con agrupamientos flexibles, donde se desarrollen propuestas curriculares individuales al estilo de los PDI (Proyectos de Desarrollo Individual), con metas personales a las que se irá realizando un seguimiento.

- Debemos aceptar y prever la evolución y el cambio de la realidad. No porque seamos adivinos/as, pues hay hechos que nadie puede escrutar, sino porque la realidad raras veces da saltos en el vacío; normalmente evoluciona y progresa. Sin ser agoreros/as, podemos prever que:

- Habrá períodos de sequía para los que nos tenemos que concienciar y el medio natural se está deteriorando a pasos agigantados. (Realidad Física). 
- La mujer tiende a igualarse en todos los terrenos y conviviremos con personas de muchas otras culturas. (Realidad Social).

- Cada vez se precisa mayor nivel de preparación para encontrar trabajo y éste cada vez es menos estable a lo largo de la vida de una persona. (Realidad Laboral).

- La informática ocupará cada vez más todos los aspectos de nuestra vida y tendremos que seguir previniéndonos del SIDA ante la ausencia de una cura próxima. (Realidad Técnica).

- Seguramente seguiremos descubriendo y estudiando estrellas y planetas lejanos mientras nos seguirá costando comprender nuestra realidad más próxima y cotidiana, sobre todo la humana. (Realidad Científica)

Queremos decir que debemos aceptar la realidad que nos rodea, aun como punto de partida para el cambio, sin perder la perspectiva de un mundo que se transforma a "pasos agigantados aunque raras veces a saltos".

Coincidimos con A. Medina cuando afirma que:

"La realidad personal, contextual y global en la que participan las PA les plantean numerosos interrogantes, que hemos de conocer $y$ adaptar a ellos las propuestas curriculares que nos propongamos." (Medina, A., 1997b: 50)

Pensamos que esos interrogantes obligan a atender, en mayor o menor medida, a la flexibilización con relación a numerosas variables, entre las que destacamos:

\section{- LA EDAD DE LAS PA}

No se debe considerar de igual modo el concepto de edad cronológica cuando somos adultos/as como se puede hacer hasta la adolescencia. Los rasgos diferenciales de los adultos van muy ligados a sus circunstancias, experiencias, compromisos y expectativas que se modifican de modo significativo con la edad, pero que se pueden segmentar en etapas determinantes con mucha mayor dificultad. En este caso como en otros, nos interesa más un mecanismo de atención a la diversidad, basado en las características de las personas, que su clasificación. Eso no debe impedirnos orientar los procesos en función de la edad -que se reflejará en una serie de características personales- en contextos específicos, por ejemplo una Escuela Taller para jóvenes.

\section{- LOS CAMPOS DE ACTUACIÓN}

En la Educación Formal, los campos de actuación suelen ir ligados al nivel de competencias instrumentales iniciales de las PA. Aunque esta valoración suele engendrar diferenciaciones de agrupamientos por niveles, no hay por qué 
preverlos y menos temporalizarlos de modo estricto en el diseño curricular. Las competencias iniciales de las PA pueden ser fruto de un proceso de desuso o puede haber factores personales motivacionales o experienciales que posibiliten un aprendizaje de ritmos muy diferentes. No queremos que se entienda que siempre lo ayudan; a veces lo frenan. El caso de muchas mujeres es ejemplificador.

\section{- LOS NIVELES DE CONOCIMIENTO}

Los niveles de conocimiento son determinantes aunque, admitiendo una posible orientación inicial sobre todo basada en las competencias instrumentales, debemos estar atentos/as a las posibilidades que ofrecen las técnicas innovadoras de diagnóstico del potencial de aprendizaje (Marín, M.A., 1987). Si podemos hacer esas consideraciones en el campo infantil y juvenil, con mayor facilidad las podremos realizar con las PA en que el triple factor personal: experiencias-compromisos-expectativas, puede ser un estimulante o una barrera significativa en el aprendizaje (véase gráfico 3 ).

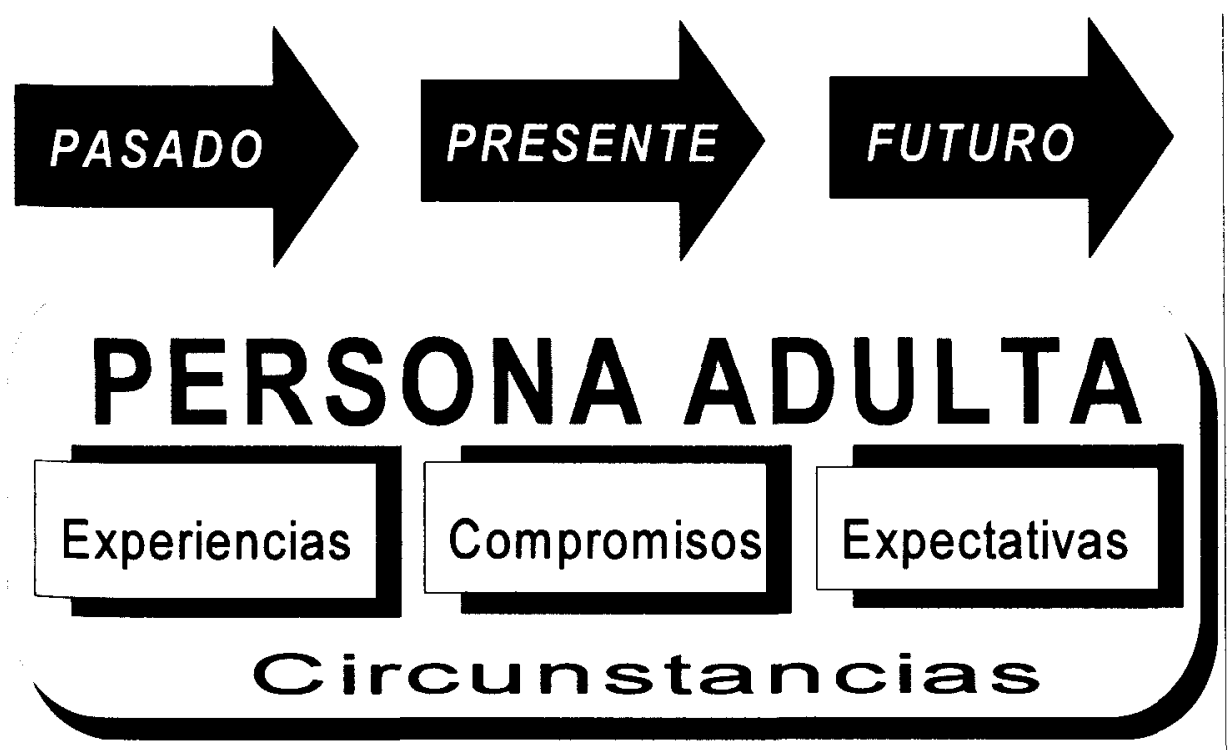

Gráfico 3. El Triple Factor Personal de la Persona Adulta: Experiencias, Compromisos y Expectativas.

\section{- LAS CARACTERÍSTICAS PERSONALES}

En general las personas tenemos características, si bien sólo consideramos con finalidades formativas las que puedan ser relevantes, determinantes o significativas a efectos de desarrollo del proceso enseñanza-aprendizaje. Entre ellas se suelen citar la autopercepción, los procesos cognitivos, los 
estilos cognitivos, las conductas de aprendizaje, las características intelectuales, el rendimiento, la adaptación, la competencia, los intereses y los valores (Jiménez Fernández, C., 1987). No obstante, no creemos suficiente que un diagnóstico en profundidad resuelva tal diversidad; sólo contando con la propia PA, favoreciendo su autoconocimiento y posibilitando sus propias elecciones personales, podemos abordar el trabajo en la práctica. Para ello contamos con personas con experiencia y autonomía, o, al menos, mucha más que en su infancia.

\section{- LOS TIEMPOS Y HORARIOS}

La flexibilidad de la temporalización es un factor clave en el aprendizaje adulto. El ritmo es un factor determinante y la causa de muchos abandonos. Podemos decir que cualquier persona "normal" puede alcanzar una meta "normal" si dispone, además de los recursos necesarios, del tiempo suficiente. Establecer de modo cerrado los momentos de evaluación final -sea trimestral, cuatrimestral o anualmente- dificulta la flexibilización del ritmo, pues obliga a una adaptación de la PA al momento de evaluación final. De este modo, establecemos un ritmo de aprendizaje a priori de sus posibilidades. Además del ritmo podemos admitir diversas variables. Resulta importante el momento: del año, de la semana y del día. La PA tiene sus compromisos: familia, trabajo, responsabilidades personales y vida social. Todo ello debería llevarnos a ofrecer momentos muy diversificados de aprendizaje.

A su vez, no estaría mal combinar esa flexibilidad con la posibilidad de disponer de las áreas, ámbitos, materias o cursos de otra forma que no sea su desarrollo simultáneo tradicional. En efecto, podemos comprobar que, por nuestro nivel de ocupación, las PA tendemos a centrarnos mejor si no llevamos tantas materias a un tiempo. En ese sentido es significativa la experiencia de los centros de Radio ECCA, antes con el Graduado Escolar, ahora con la experimentación del GES. Como se ve, los centros a distancia también tendrían mucho que pensar sobre la flexibilización del tiempo. A la vez, deberíamos realizar una reflexión sobre el paso del tiempo y nuestro aprendizaje con algunas preguntas:

¿Cuánto perduran nuestros aprendizajes? ¿Cómo los actualizamos? ¿Cómo podemos aprender a mantener lo que aprendemos? ¿Debe formar parte de una titulación básica un saber caduco? ¿Qué es lo perenne?

\section{- LOS ESPACIOS Y LUGARES}

El espacio puede ser entendido como contexto. En ese caso, los procesos de enseñanza-aprendizaje deben estar contextualizados. El espacio contextual no es sólo un espacio físico sino también simbólico, ideológico, cultural, habitual y vivencial. Por ello, la contextualización es un proceso construido de modo participativo y democrático. Debemos aceptar la PA que tenemos delante como admitimos la realidad que nos rodea. Aunque propongamos 
metas liberadoras o concienciadoras, sólo serán lícitas si son compartidas y aprobadas por la persona en formación.

En cuanto a los lugares, tendremos que acercar lo mejor posible los procesos de formación a las PA. Ello no debe llevarnos a fomentar la precariedad tan habitual en algunas instalaciones para la FPA, sino a desarrollar políticas de creación de infraestructuras integradas en instalaciones polivalentes y versátiles que favorecerán su rentabilización y su adecuación. La idea de las "casas del pueblo" o de las "casas de la cultura" podría incentivar esta red pluridimensional para usos formativos, culturales, participativos y comunitarios.

Ello nos avoca a considerar que:

“(..) la educación de adultos no sea un campo de transferencia y adaptación de otros niveles del sistema educativo, sino un espacio propio que configura sus bases y sentido, proyectando sus hallazgos y exigencia a otros niveles del Sistema Educativo." (Medina, A. y Domínguez, C., 1995:95).

Si aceptamos este reto, afrontaremos una nueva concepción del currículum. La flexibilización será una pieza clave para ello. En este sentido aportamos un esquema-marco (véase gráfico 4) para plantear, revisar o/e implementar las propuestas curriculares para las PA. Para ello proponemos una revisión de los siguientes puntos:

\begin{tabular}{|l|}
\hline - Estructura del currículum \\
- Organización del conocimiento \\
- Niveles de referencia \\
- Proceso de diseño \\
- Contenidos \\
- Desarrollo de la práctica \\
- Evaluación
\end{tabular}

No es posible concebir la flexibilización de los procesos formativos de la enseñanza obligatoria con los mismos parámetros que en la enseñanza no obligatoria. Por ello no nos debe sorprender que cuestionemos lo que podría resultar paradigmático en la enseñanza obligatoria, como la organización del conocimiento o los niveles de referencia. Ni los determinantes o condicionantes ni las posibilidades y capacidades de la PA son óbice para ello. Más bien debemos esforzarnos en establecer un marco creativo para el trabajo con PA que pueda dar lugar a propuestas curriculares divergentes y diferenciadas. 


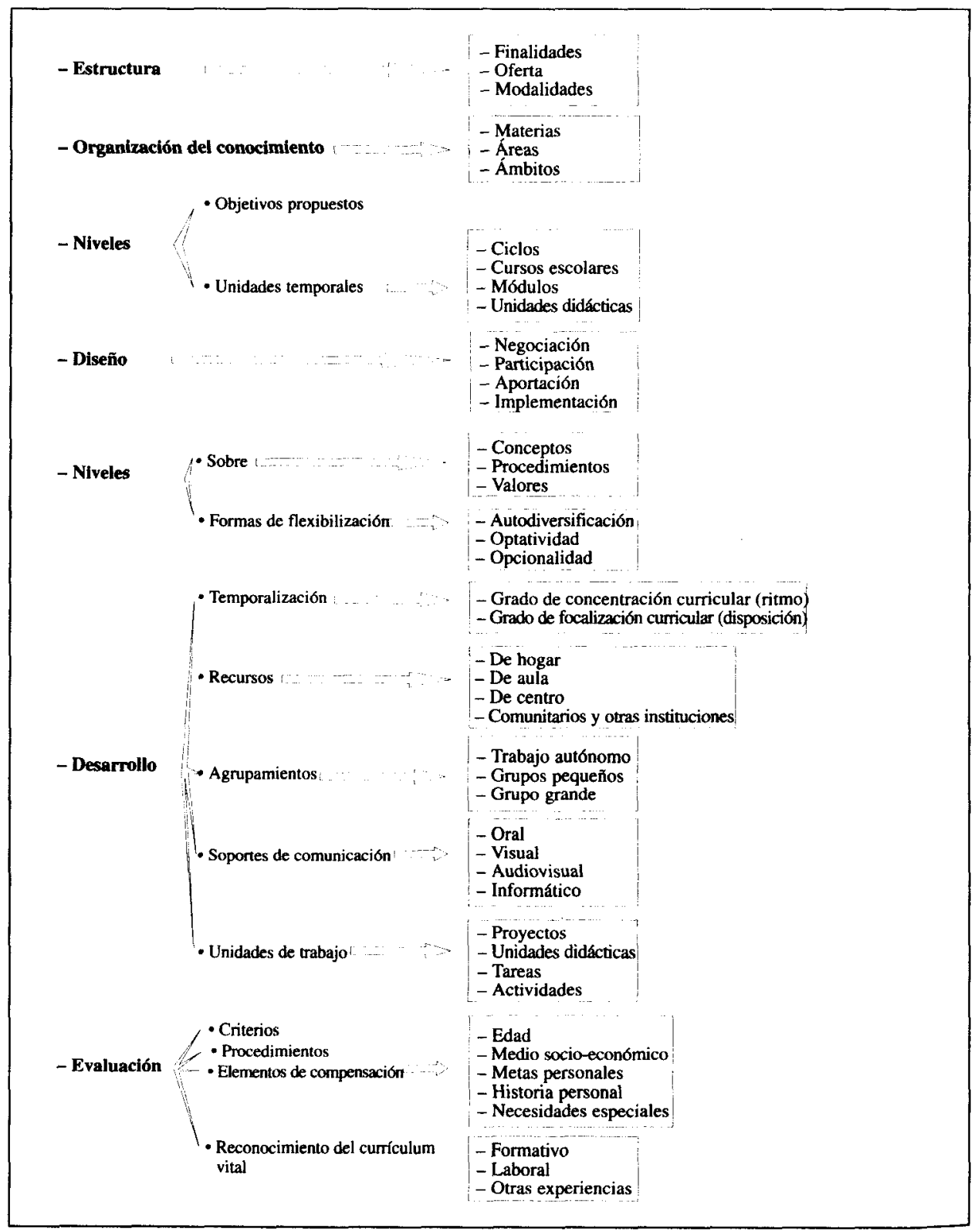

GrÁfICo 4. Algunos modos de flexibilización del currículum.

\section{A. ESTRUCTURA DEL CURRÍCULUM}

La estructura del currículum no tiene por qué dársenos cerrada. Antes bien sería deseable que se nos ofreciera abierta e indefinida. ¿Es realmente preciso? Pongamos algún caso: 
¿Sería lícito plantearnos un diseño curricular de GES para personas que deseen acceder al Bachillerato? ¿O al curso de acceso a la universidad para mayores de 25 años? ¿O a un módulo/curso/programa formativo de carácter profesional? ¿O simplemente para ser una persona que se desenvuelva con facilidad en su medio? ¿Acaso pensamos que garantizaríamos un mayor cumplimiento de unos supuestos "mínimos" por establecerlos con carácter prescriptivo estructurados con sus objetivos, conceptos, procedimientos y valores, y sus criterios de evaluación?

Desde este punto de mira, debería ser posible alterar, cambiar, negociar y/o replantear las mismas finalidades formativas de un GES. Por este camino, es fácil imaginar que se acrecentaría asimismo la variedad de ofertas y de modalidades formativas, sobre la base de propuestas diferenciadas integradas en/con otras propuestas formativas, con parámetros y valores diferenciados, singulares y plurales, repletas de vida y de riqueza. ¿No podemos entender que podemos ser personas formadas y ciudadanos/as responsables de mil y una formas?

\section{B. ORGANIZACIÓN DEL CONOCIMIENTO}

La realidad es global. Lo que no quiere decir que todo tiene que ver con todo, sino que las relaciones que se mantienen no tienen por qué ver con la lógica de nuestro conocimiento. El conocimiento más primario e intuitivo que aplicamos es, de hecho, holístico. Tampoco significa que lo percibimos todo. Percibimos en función de nuestros intereses, de nuestras motivaciones, determinadas, como hemos visto, por nuestro pasado, nuestro presente y nuestro futuro, o por lo que nos imaginamos de ellos. Por ello una propuesta curricular tiene que poder articularse de modo que resulte efectiva -motivadora, formativa, movilizadora- y ello sólo será realidad si podemos organizar el conocimiento en base a los parámetros que establezcamos con las PA en formación. No discutimos que éstas sean más o menos puntuales o estables, sino que tienen que poder ser implementadas o transformadas radicalmente. Si en un centro, con un alumnado determinado, en un momento dado, funciona adecuadamente un sistema modular de unidades capitalizables, jadelante! Pero ello no tiene que llevarnos a elevar a rango de norma universal el modelo. Ni siquiera en ese mismo centro: en otro momento, otro profesorado, otro alumnado, por lo que sea, obtiene mejores resultados con otra organización del conocimiento. Por ello la discusión sobre la organización en materias, áreas o ámbitos, o cualesquiera que sean, resulta baldía. Inclusive, si a efectos de currículum prescriptivo se partiese de un determinado modelo, se tendrían que posibilitar otros desarrollos organizativos alternativos, sin perjuicio de su equivalencia para la obtención del GES.

\section{NIVELES DE REFERENCIA}

Solemos organizar nuestra oferta en niveles. Sirven de referencia al alumnado para situarse en su recorrido formativo, al profesorado a efectos 
organizativos y a la administración con el fin de la titulación. La misma idea de niveles conlleva graduación y secuenciación, pero podrían darse éstas sin niveles. Los objetivos propuestos pueden concebirse como un continuo, a semejanza de una escalera. Los pisos no deben ser en modo alguno fronteras y barreras. Antes bien posibilitar la continuidad es un elemento de mayor interés que el paso de controles aduaneros. Nuestras aulas tienen a veces demasiado el aspecto de tanques herméticos e indivisibles; y nuestros cursos escolares también. De ahí que el establecimiento flexible de unidades temporales sea un pilar importante para posibilitar un acceso democrático a la formación. No todos/as podemos siempre y en cualquier momento. Ello significa que debemos promover ofertas singulares y olvidarnos del curso escolar como unidad intocable. Estas unidades tienen que posibilitar un recorrido formativo progresivo y continuo, y evitar constituirse en células impermeables y cerradas. Así mismo debemos posibilitar la entrada y la salida del alumnado a lo largo del proceso, para respetar su derecho a la formación y reconocer sus logros y su trabajo de aprendizaje. El establecimiento en niveles debería ser, pues, un elemento diseñable en cada situación concreta, admitiendo incluso su ausencia por completo. Otras alternativas podrían ser los ciclos, los cursos escolares, los módulos o las unidades didácticas.

\section{EL PROCESO DE DISEÑO}

En un sistema abierto y flexible en el que, además, el alumnado tiene una gran movilidad, se imponen otras formas de diseño curricular. Si a ello sumamos que las PA tiene también sus criterios personales y su capacidad para opinar y realizar aportaciones valiosas, se nos ofrecen unas posibilidades insospechadas en otros contextos. Nos referimos a que los procesos de elaboración deben engendrar dinámicas de negociación, participación, aportaciones -personales, grupales y/o comunitarias-, así como todo tipo de implementaciones compartidas. Las dinámicas que se engendren al amparo de estas premisas resultarán enriquecedoras y formativas en sí mismas, pues plantearán el desarrollo del conocimiento propio y mutuo, de la toma de decisiones, de la aceptación de responsabilidades y el hábito de la previsión. En cualquier caso, un proceso compartido en el diseño también lo será en su asunción y en la responsabilidad de su desarrollo y de sus resultados.

\section{E. CONTENIDOS}

La flexibilidad de los contenidos puede afectar a sus 3 dimensiones: conceptual, procedimental y valoral. Pero no tenemos que enfatizar ni darle supremacía al aspecto conceptual de los contenidos. Pensemos en nosotros/as mismos/as y observemos cómo nos hacemos selectivos: nuestros gustos, nuestras necesidades y nuestras obligaciones van delimitando nuestros conocimientos, nuestras habilidades y nuestras preferencias. Y no podría ser de otro modo. ¿Por qué empeñarnos en no aceptar esa diversidad en nuestro alumnado? ¿Por qué no aceptar que una PA pueda saber más, hacer mejor o preferir una materia curricular sin perjuicio de que 
sepa menos, haga peor o no valore otras? ¿Acaso no nos sucede a todos/as? Se podría plantear el problema del carácter propedéutico de la enseñanza. Creo que ese aspecto debe quedar al amparo de la responsabilidad de la propia PA. Si realmente piensa continuar estudiando, haría bien en orientar su proceso formativo con ese carácter y deberemos ayudarle a comprender esa necesidad.

Para facilitar la flexibilización de los contenidos debemos introducir nuevos conceptos en nuestro marco de GES como:

- La autodiversificación por la que la PA plantea y asume modificaciones de su recorrido formativo.

- La optatividad mediante la cual planteamos la elección entre diversas alternativas.

- La opcionalidad con la que proponemos partes voluntarias en el proceso formativo.

\section{F. DESARROLLO}

Las premisas que establezcamos para el desarrollo de nuestra práctica formativa son de suma importancia para la flexibilización real del proceso. En ello juegan gran importancia:

\section{- La temporalización}

Nos pararemos en este momento en 2 variables que nos resultan de gran interés. Por una parte, el grado de concentración curricular, es decir, la velocidad, el ritmo, la diligencia con que avanzamos en nuestro proceso de formación. Debemos respetar los ritmos personales porque son una característica del modo de aprendizaje de cada cual y porque es uno de los pilares que garantiza la permanencia en el proceso de formación. Si "no se sigue" el ritmo, difícilmente se puede alcanzar ninguna meta. Que nos siga el ritmo a nosotros/as. Es más fácil y consolida el autoconcepto al tiempo que refuerza la autoestima. Por otro lado, nos parece de interés el grado de focalización curricular, es decir, la disposición de los focos de aprendizaje. Los focos de aprendizaje están constituidos por los núcleos que merecen nuestra atención en un momento determinado. Nos resulta más fácil centrarnos en un número asequible de focos que dependerá con seguridad de cada persona. Por ello, no deberíamos ignorar la posibilidad de reducir el número de focos de aprendizaje, y algunos modos de organización del conocimiento o del establecimiento de unidades de trabajo lo facilitan a la vez que suelen propiciar una formación más significativa. No obstante, como hemos apuntado con anterioridad, ciertas experiencias han puesto de relieve que incluso una organización modular y de áreas de formación como teníamos en graduado escolar se pueden organizar en torno a menos focos, por ejemplo: reduciendo las áreas de formación que se realizan de modo simultáneo. 


\section{- Recursos}

Los recursos didácticos también admiten todo tipo de planteamientos en la naturaleza, la procedencia, la presentación o la utilización. Deberíamos aprender a aprovechar la inmensidad de recursos que acumulamos en nuestros hogares (un recibo de electricidad o un folleto publicitario son excelentes recursos), así como los recursos comunitarios y de otras instituciones. El propio medio es un recurso increíble. Para ello tenemos que perder el miedo a salir del aula y contar con nuestro alumnado. Ello no será impedimento para que contemos con recursos de aula y del centro, así como con los que podamos encontrar en centros de recursos institucionales o comunitarios específicamente destinados a los centros formativos.

\section{- Agrupamientos}

El trabajo a distancia suele realizarse de forma autónoma, aunque no tiene por qué ser así. De hecho, la constitución de grupos de estudio y de autoapoyo resulta de gran interés para la permanencia en procesos de formación a distancia. Si bien tenemos que plantear la dificultad con que nos enfrentamos cuando intentamos organizar el trabajo en agrupamientos diferentes. No obstante, la experiencia es rica en muestras de planteamientos como el trabajo autónomo, los grupos pequeños o los grupos grandes en dinámicas interactivas y participativas. Es difícil una individualización que no pase por una reorganización de los procesos de aprendizaje en el aula... y fuera de ella. Como en todo, la variación facilitará la motivación. Debemos evitar el "dinamiquismo" o exceso sin sentido, pero pocas veces se nos dará el caso.

\section{- Soportes de comunicación}

En los procesos a distancia resulta una variable evidente, pero en el aula también. En los procesos autónomos de aprendizaje los soportes tienen que facilitar la adaptabilidad y el seguimiento por la PA. En los procesos dirigidos son buenos recursos que deben estimular la motivación y la compresión. Los soportes orales/auditivos son más intuitivos que los escritos; los visuales, particularmente los audiovisuales, son así mismo muy atractivos. Pero hay que reconocer que en el mundo en que vivimos, los países desarrollados de la Europa Comunitaria, resulta muy difícil motivar con la imagen. Estamos muy acostumbrados a ver los efectos especiales más espectaculares como para sorprendernos con cualquier filmación. En ese caso, deberíamos recordar que "raras veces la ficción supera a la realidad". Finalmente señalar que, si bien es cierto que la informática se apodera cada vez más de nuestra vida, no todos/as podemos disponer de ordenador por ahora, ni por algún tiempo. Mientras, cualquiera tiene una radio. Debemos considerar estos aspectos cuando pensamos en el derecho a la educación antes que en el reconocimiento personal.

\section{- Unidades de trabajo}

Nuestro trabajo se puede articular en unidades de trabajo muy diversas que hemos esbozado, pues conllevan de hecho modos diferenciados de 
organización del conocimiento. Cuando hablamos de unidades de trabajo, estamos pensando en las formas de aprendizaje más inmediatas que percibe nuestro alumnado. Hemos oído hablar de unidades didácticas, de tareas o simplemente de actividades con todas sus variantes: ejercicios, problemas, proyectos, prácticas, cuestiones, preguntas, etc. Variar nuestros planteamientos didácticos puede enriquecer sumamente nuestra acción formativa.

\section{G. EVALUACIÓN}

Finalmente nos adentraremos, aunque superficialmente, en los procesos de evaluación. Si reivindicamos la flexibilidad y la diversidad, tendremos que asumir que los criterios tienen que quedar abiertos para poder ser individualizados para cada persona. Los planteamientos apuntados al estilo de Proyecto de Desarrollo Individualizado presuponen esa posibilidad. No se puede exigir a todos por igual. Y de hecho no lo hacemos. ¿Por qué no reconocerlo? No tratamos por igual a una mujer de 70 años que a un joven de 18. No exigimos por igual a la persona que necesita la titulación con fines propedéuticos a la que la precisa para trabajar. ¿Por qué nos cuesta tanto reconocerlo y aceptarlo? La justicia no es dar a todos/as por igual. Ello nos obligaría a explicitar los elementos de compensación que estamos dispuestos a asumir: la edad, el medio socio-económico, las metas personales, la historia personal o incluso las necesidades especiales. Los procedimientos son así mismo un elemento a flexibilizar pero es muy importante la concepción que tengamos de la evaluación. Insistiremos simplemente en que debe facilitar un recorrido formativo continuo sin saltos ni barreras artificiales.

Al tratarse del GES, no podíamos omitir la posibilidad del reconocimiento de otros elementos, como ya hemos apuntado anteriormente. El currículum vital de una persona está constituido por su experiencia formativa, su recorrido laboral, además de otras experiencias de difícil clasificación. Deberíamos estar abiertos/as al reconocimiento de todo ello, pero sin vulnerar los más básicos de la orientación: el reconocimiento no puede suplir a destrezas o habilidades si no las tiene. Es decir, no puede dar acceso a una formación si con ello omitimos fases formativas necesarias para continuar. Flaco favor le haríamos. Ello no nos debe impedir buscar fórmulas adecuadas (cuotas, porcentajes, créditos, etc.) que posibiliten el reconocimiento de elementos tan valiosos como los que adquieren en nuestras aulas. A veces incluso más valiosos.

En definitiva, consideramos que el porvenir pasa irremediable y dichosamente por la diversificación y la flexibilización que posibilitarán, en gran medida, las transformaciones cósmicas pertinentes que estamos obligados/as a afrontar, aunque cada Persona Adulta tendrá que recorrer su propio camino. Éste no lo haremos solos/as. Lo realizaremos en compañía con un margen humanamente lícito de maniobra. Como profesionales nos queda el reto de saber plantear nuestro trabajo en la diversidad y la flexibilidad. Debemos indagar para aprender a crear las condiciones que, respetando la primera, posibiliten la segunda. 


\section{BIBLIOGRAFÍA}

ALONSO, A. (coord.) (1995). Hacia un nuevo contrato educativo: La educación de adultos. Un plan para la Rioja. La Rioja, Gobierno de la Rioja.

GÓMEZ DE CASTRO, F. (1988). “Los sistemas educativos y la revolución francesa". GÓMEZ DE CASTRO, F., RUIZ BERRIO, J., OSSENBACH, GUTIÉRREZ, A., TIANA, A. (coords.). Génesis de los sistemas educativos nacionales. Madrid, UNED. Pp. 17-39.

FLECHA, R, LÓPEZ, F. y SACO, R. (1988). Dos siglos de educación de adultos. Barcelona, Roule.

FLECHA, R. (1990). Educación de las personas adultas: Propuestas para los años noventa. Barcelona, Roule.

FELIZ MURIAS, T. y RICOY LORENZO, M.C. (1996). "La interacción comunicativa en la formación de personas adultas". ROSALES, C. (coord.). Actas del I Congresos de innovación educativa. Santiago de Compostela, Tórculo edicións. Vol. II, pp. 431-440.

FERRÁNDEZ, A. y PEIRÓ, J. (coords.) (1989). Modelos formales y no formales en educación de adultos. Barcelona, Humanitas.

FERRÁNDEZ, A. y PEIRÓ, J. (coords.) (1989). Métodos y técnicas en la educación de adultos. Barcelona, Humanitas.

GENERALITAT VALENCIANA (1993). Diseño curricular para la formación básica de las personas adultas en la comunidad valenciana. Valencia, Generalitat Valenciana.

GONZÁLEZ SOTO, A. (1989). Formación de adultos. Un modelo de unidades modulares. Barcelona, Humanitas.

JARVIS, P. (1989). Sociología de la educación continua y de adultos. Barcelona, Roule.

JIMÉNEZ FERNÁNDEZ, C. (1987): Cuestiones sobre bases diferenciales de la educación. Madrid, UNED.

LEIRMAN, W. (1994). "El futuro de la educación de adultos en Europa: Un proyecto de investigación de tipo
Delphi en 14 países". REQUEJO, A. GONZÁLEZ, A. y LETE, J.R. (coords.). Educación de adultos en una sociedad en transformación. Pontevedra, Xunta de Galicia. Pp. 87-103.

LEY ORGÁNICA 1/1990, DE 3 DE OCTUBRE, DE ORDENACIÓN GENERAL DEL SISTEMA EDUCATIVO. BOE, núm. 238, de 4 de octubre.

MARIN, M.A. (1987). El potencial de aprendizaje. Barcelona, PPU.

MEC (1986). Educación de adultos. Libro Blanco. Madrid, MEC.

MEDINA RIVILLA, A. (coord.) (1995). Formación de educadores de personas adultas II. Madrid, UNED. Tomo I.

MEDINA RIVILLA, A. (coord.) (1997a). Diseño y desarrollo curricular para la formación de personas adultas. Madrid, UNED.

MEDINA RIVILLA, A. (1997b). “Diseño curricular básico para personas adultas en el humbral del siglo XXI". MEDINA RIVILLA, A. (coord.). Diseño y desarrollo curricular para la formación de personas adultas. Madrid, UNED. 29-57.

MEDINA, A., ALONSO, C., DOMÍNGUEZ, C., DOMINGUEZ, G., GALLEGO, D., GENTO, S., IBAÑEZ, A. y TORRIJOS J. (1995). Formación de educadores de personas adultas I. Madrid, UNED.

MEDINA, A. y DOMINGUEZ, C. (1995). Enseñanza y curriculum, para la formación de personas adultas. Madrid, EDIP.

PÉREZ SERRANO, G. (1994): Investigación cualitativa. Retos e interrogantes. I Métodos. Madrid, Muralla.

PÉREZ SERRANO, G. (1994): Investigación cualitativa. Retos e interrogantes. II Técnicas y análisis de datos. Madrid, Muralla.

RICOY LORENZO, M.C. (1994): “Necesidad del reconocimiento y potenciación de la educación no formal de las personas adultas". REQUEJO, A. GONZÁLEZ, A. y LETE, J.R. (coords.). 
Educación de adultos en una sociedad en transformación. Pontevedra, Xunta de Galicia. Pp. 219-226.

RICOY LORENZO, M.C. (1996): El curriculum de las personas adultas en educación no formal. Santiago de Compostela, Tórculo edicións.

RICOY, M.C. (1997). “La prensa en la educación de personas adultas". Revista Reccamara, (O), 20-22.

RICOY, M.C. y FELIZ MURIAS, T. (1997). "Multifuncionalidad de la informática: análisis de las propuestas didácticas actuales en la formación de personas adultas". ALONSO, C y GALLEGO, D. (coords.). La informática desde la perspectiva de los educadores. Madrid, UNED. Vol. II, pp. 119-128.
TENNANT, M. (1988): Adultez y aprendizaje. Barcelona, Roule.

SARRAMONA, J. (1989): "Educación de adultos por metodología no formal". FERRÁNDEZ, A. y PEIRO, J. (coords.). Modelos formales y no formales en educación de adultos. Barcelona, Humanitas. Pp. 31-41.

SEPÚLVEDA, F. (1997): "La comunicación, base del currículum para personas adultas. El diseño curricular de lengua”. MEDINA, A. (coord.). Diseño y desarrollo curricular para la formación de las personas adultas. Madrid, UNED. Pp. 251-271.

YUS RAMOS, R. (1997): Hacia una educación global desde la transversalidad. Madrid, ALAUDA-ANAYA.

\section{RESUMEN}

Los retos del siglo XXI en Educación Secundaria para Personas Adultas pasan por acrecentar las vías de diversificación y flexibilización con relación a diferentes aspectos: edad, campos de actuación, niveles de conocimiento, caracteristicas personales, tiempos y horarios, espacios y lugares, currícula, etc. La Formación de Personas Adultas tenderá a ampliarse y consolidarse en el próximo milenio, ello posibilitará las adaptaciones y transformaciones constantes en una sociedad de cambio permanente.

La Formación de Base para Personas Adultas pasa por contribuir a la madurez personal y socio-laboral de las mismas, ayudándoles en su actualización desde una perspectiva lo más holística posible de la realidad como ente global que camina hacia la globalización y mundialización del conocimiento. Éste facilitará la movilidad ciudadana en la Comunidad Europea. La Formación de Personas Adultas tenderá a aumentar su oferta en función de la previsible creciente demanda de actualización permanente, aumentando la implicación en relación con la cantidad de instituciones, empresas y grupos que oferten programas de formación, especialmente en el campo de la Educación no Formal. Creemos y deseamos que la Formación Permanente -y, por supuesto, en ella está incluida la Formación de Personas Adultas-se consolidará y tenderá a garantizarse en el próximo milenio. 
Palabras clave: Currículum. Diversificación. Educación/Formación de Personas Adultas. Graduado en educación secundaria. Flexibilización. Futuro de la educación permanente.

\begin{abstract}
The challenges of the XXI century in Secondary Education for Adult People (AP) are to increase the ways for diversification and for making it more flexible in different aspects: age, performance fields, levels of knowledge, personal characteristics, times and timetables, spaces and places, curricula, etc. Adult education will tend to enlarge and consolidate in the next millenium, this fact will ease adaptations and constant transformations in a society of continuous change.

The Basic Learning for AP goes to contribute to their personal and social maturity, helping them in their updating from the most holistic possible perspective of reality as a global entity that walks towards globalization and worldwide extension of knowledge. This will ease people mobility throughout the European Community. The AP learning will increase its offer according to the foregone growing demand of permanent updating, increasing the implication especially in relation to the quantity of institutions, companies and groups that offer learning programs, in the field of the non Formal Education. We believe and we wish that permanent education-considering adult education included in that concept-will consolidate and it will tend to warrant itself in the next millenium.
\end{abstract}

Key words: Adult People. Basic diploma in Secondary education. Curriculum. Diversification. Education and learning of adult people. Future of permanent education. Secondary education. 